\title{
The vegetative state
}

\section{B Jennett}

\section{The definition, diagnosis, prognosis and pathology of this state are discussed, together with the legal implications}

d n the 30 years since this state was first described and named ${ }^{1}$ it has provoked intense debate not only among clinical scientists and health professionals but also among moral philosophers and lawyers. Considering its relative rarity there is also, courtesy of the media, an unusua degree of public awareness of the condition. What attracts attention and curiosity is the dissociation between arousal and awareness-the combination of periods of wakeful eye opening with lack of any evidence of a working mind either receiving or projecting information. The advantage of the term "vegetative state" is that it simply describes observed behaviour, without implying specific structural pathology. However, since the realisation that this state is frequently temporary, the original term persistent vegetative state is potentially misleading as it suggests irreversibility. After a certain length of time it may none the less be reasonable to describe this state as permanent. ${ }^{2}$

Before specific diagnostic criteria were agreed, some reports had used less rigorous definitions. ${ }^{3}$ The most widely accepted criteria are those in the 1994 report from the US Task Force. ${ }^{4}$ These are essentially negative-the lack of evidence of awareness of the self or the environment, of interaction with others, or of comprehension or expression of language. By implication, external stimuli do not evoke purposeful or voluntary behavioural responses that are sustained and reproducible. There are two problems in applying these criteria. One is the wide range of reflex responsiveness in some vegetative patients, some of which can give rise to suspicion of meaningful mental activity. The other is the limited range of voluntary responses available to severely brain damaged patients, which can make the detection of awareness difficult. ${ }^{5}$ The recent publication of detailed practical tests for detecting that a patient is in a minimally conscious state rather than vegetative should help to reduce the risk of misdiagnosis. ${ }^{67}$

The simplistic assertion that vegetative patients can be defined as showing no sign of cerebral cortical function has fuelled debate as to whether certain behaviours entail any cortical activity.
More important, however, is whether there is evidence of sufficient cortical integrity to indicate awareness-which is the crux of the definition. Such integrity is not required for sudden light or sound to stimulate a startle response or an orienting reflex with the head and eyes turning briefly towards the stimulus. Whether blink to visual threat need imply limited cortical activity is debatable, but this response is not itself evidence of awareness. Because visual pursuit is usually one of the first observable signs of recovery in patients who go on to regain consciousness, some consider that patients who show this phenomenon are no longer vegetative. However, several authorities maintain that occasional vegetative patients do regain some capacity for visual pursuit without developing any other behavioural evidence of consciousness during the following months or years. The Task Force concluded: "One should be extremely cautious in making a diagnosis of the vegetative state when there is any degree of sustained visual pursuit or consistent and reproducible visual fixation." ${ }^{\prime 4}$ The Royal College of Physicians criteria in 1996, which seemed to be designed to exclude cortical activity rather than showing lack of awareness, list "no nystagmus to caloric testing, no visual fixation, tracking, or response to menace" under the heading 'Other criteria'. ${ }^{2}$ In several cases since then the English High Court has chosen to accept expert opinion that particular patients in whom one or more of these eye responses were active were indeed unaware and therefore vegetative.

The spastic limbs may move in a nonpurposive way, including groping and grasping. Noxious stimuli may provoke limb withdrawal that may be associated with facial grimacing and a rise in respiratory and pulse rates and in blood pressure; these do not in themselves indicate conscious experience of pain. Some vegetative patients may occasionally smile or frown, and less often laugh or weep, but these emotional behaviours show no consistent relation to an appropriate stimulus. Intermittent episodes of complex behaviour have been reported in three American patients who were vegetative by all other criteria, and whose global brain metabolism level was below $50 \%$ of normal. ${ }^{8}$ One patient, 20 years vegetative, uttered single words from a vocabulary of four or five once every two or three days. Another, seven years vegetative, responded to loud noise or attempts at nursing care by clenched teeth, rigid extremities, and high pitched screaming that abated in response to soothing voices or music. All three patients had limited areas of cortex that showed metabolic levels that were higher than elsewhere although still well below normal. The conclusion was that these patients had isolated segregated cortical networks that retained connectivity and partial functional integrity. In two other vegetative patients without unusual behaviours this team observed some preserved cortical metabolism; in one case this was virtually normal throughout the brain but there was severe bilateral thalamic damage. Others have recorded evidence of residual cortical activity in vegetative patients, ${ }^{9}$ and there has been an exchange of views between these two laboratories about the significance of their findings. ${ }^{10}$ Some patients diagnosed as permanently vegetative have had repeated episodes of tonic-clonic epilepsy, further evidence of residual cortical activity.

The dominant lesions in the brains of many vegetative patients are thalamic and subcortical white matter damage. ${ }^{11}$ Of 35 traumatic cases $80 \%$ had thalamic damage and $71 \%$ severe diffuse axonal injury; in seven the cerebral cortex was normal and in 21 there were only minor cortical contusions. By contrast study of the brains of 30 cases severely disabled after head injury found that half had neither severe diffuse axonal injury nor thalamic damage, a negative combination found in not a single traumatic vegetative case. $^{12}$ Of 14 non-traumatic vegetative cases only $64 \%$ had diffuse cortical damage and one had a completely normal cortex, but every case had severe thalamic damage. ${ }^{11}$ The conclusion is that consciousness depends on the integrity of sufficient thalamocortical and intercortical connections, and that isolated neural activity involving parts of the cortex, even when associated with minimal stereotyped behavioural expression, need not indicate even minimal consciousness. However, much remains to be understood regarding the mechanisms underlying the vegetative state.

Estimates of the frequency of the vegetative state vary widely because cases are difficult to locate in many different sites of care, and because of variations in how long patients need to have been vegetative for inclusion in a survey. This can vary from one to six months, one month being specified by the Task Force. American prevalence figures, including those resulting from chronic conditions, vary from 64 tol 40 per million population (PMP), about one third of them 
children. ${ }^{13}$ The annual incidence from acute causes varies in different countries from 14 to 67 PMP at one month after the insult but only 5 to 25 PMP at six months, with the UK figures being the lowest. ${ }^{3}$ This reflects the lower incidence of severe head injuries in the UK, as trauma accounts for $40 \%-50 \%$ of vegetative survivors after acute insults.

The best data available on the prognosis of vegetative patients are from the analysis of 754 published (acute) cases by the Task Force. ${ }^{4}$ Of those vegetative at one month $43 \%$ had regained consciousness by one year, $34 \%$ were dead, and only $23 \%$ were still vegetative. The longer the vegetative state had lasted the fewer recovered-after six months only 13\% regained consciousness. Trauma cases did better (table 1), and head injured children did marginally better than adults. It is because of the attrition by death and recovery that the incidence and prevalence rates reduce as time passes since the insult. There are few systematic data on cases followed up for more than a year but the Task Force decided that it was reasonable to declare the vegetative state permanent after one year in traumatic cases and after three months in the non-traumatic. However, the Royal College of Physicians report recommended six months for the latter cause. ${ }^{2}$ There are occasional reports of late recoveries, more in the public media than are medically verified. It is, however, important to realise that many patients who regain consciousness after several months remain speechless and tube fed with very limited ability to communicate. To state that these patients have "recovered" may seem to some a rather optimistic claim. Some of them are in the minimally conscious state, $^{6}$ showing some responses that indicate a limited degree of awareness. However, the wisdom of attempting to define such a state has been questioned by disability rights campaigners in America. ${ }^{14}$

There is a high mortality during the first year and the Task Force reported a mean survival of only two to five years for patients vegetative at one month. What is of more interest, however, is the expectation of life for those who have already survived in a vegetative state for a year or more. The continuing annual mortality then reduces year by year, and there have been occasional survivals of 20 or more years. A systematic study in California drew attention to the difference between mean and median survival, the latter being shorter because not distorted by the occasional very long survival. ${ }^{15}$ For a young adult vegetative after one year this study calculated a mean survival of 10.5 more years, the median being 5.2 years. For one still vegetative four years after the insult these figures increase to 12.2 and 7 more years respectively.

Table 1 Outcome one year after insult according to duration of the vegetative state

\begin{tabular}{lllll}
\hline & Number & Dead & Vegetative & Conscious \\
\hline Vegetative after one month & 540 & $28 \%$ & $18 \%$ & $54 \%$ \\
$\quad$ Traumatic & 214 & $47 \%$ & $39 \%$ & $14 \%$ \\
$\quad$ Non-traumatic & 268 & $31 \%$ & $30 \%$ & $39 \%$ \\
Vegetative after 3 months & 108 & $36 \%$ & $58 \%$ & $6 \%$ \\
$\quad$ Traumatic & & & & \\
$\quad$ Non-traumatic & 151 & $28 \%$ & $53 \%$ & $19 \%$ \\
Vegetative after 6 months & 80 & $18 \%$ & $81 \%$ & $1 \%$ \\
$\quad$ Traumatic & & & \\
$\quad$ Non-traumatic & & & \\
\hline Derived from Task Force data. ${ }^{4}$ & & &
\end{tabular}

In the early months it is important that everything is done to maintain the patient in the best possible general condition so that the most can be made of any spontaneous neurological recovery that may occur. ${ }^{16}$ Good nutrition is essential to maintain body weight, usually via a percutaneous endoscopic gastrostomy, and joint mobility should be preserved to minimise contractures. Regimens of sensory stimulation (so called coma arousal programmes) have been advocated but there is no good evidence that these promote the recovery of consciousness. ${ }^{17}$

The ethical and legal debates focus on attitudes to patients declared permanently vegetative. There have been many declarations that survival in a permanent vegetative state is not a benefit to the patient, some regarding it as a fate worse than death. Numerous surveys of the attitudes of patients, doctors, nurses, and ethicists have confirmed that this is a widespread view, with many respondents indicating that they would not want life prolonging treatment if in this state. ${ }^{3}$ At the same time there is increasing concern in medical ethics for respecting patient autonomy when making decisions about treatment. Competent patients have an absolute right to refuse the initiation or continuation of any treatment, even when this is life prolonging, if they regard it as bringing more burdens than benefits. The problem is that vegetative patients are not competent to refuse continued treatment, and there is concern about how best to protect them from treatment that they would probably refuse if they could. Few patients have made an advance directive and there has been much debate about appropriate surrogate decision makers. In the US families are allowed to assume this role but in the UK only a doctor may make such a decision about an incompetent adult patient, albeit after discussion with the family. While it is easy enough to decide to withhold cardiopulmonary resuscitation or antibiotics for acute infections, many vegetative patients survive for years after a decision to limit these treatments. The question then arises of withdrawing artificial nutrition and hydration-regarded by most authorities as a form of medical treatment. There is no moral or legal obligation for a doctor to provide a treatment that is not in the best interests of his patient. Although some object that such withdrawal might seem to condone euthanasia this has been challenged. ${ }^{18}{ }^{19}$ A recent review of the outstanding ethical issues in the UK notes the need to consider also the question of justice in allocating scarce resources to the indefinite support of vegetative patients. ${ }^{20}$ In many common law jurisdictions it is now agreed that withdrawal of life sustaining treatment is lawful, ${ }^{321}$ and an English judge recently ruled that it does not infringe the European Convention on Human Rights. ${ }^{22}$ Only in the UK, however, is High Court approval still required before taking such action, and some lawyers do not consider that the legal situation has yet been satisfactorily resolved. ${ }^{23} 24$

$J$ Neurol Neurosurg Psychiatry 2002;73:355356

\section{Authors' affiliations}

B Jennett, Department of Neurosurgery, Institute of Neurological Sciences, Glasgow, UK

Competing interest: BJ has appeared as an expert witness in the High Court when a declaration was sought that withdrawal of treatment from a number of specific patients in the permanent vegetative state would be lawful.

Correspondence to: Professor B Jennett, 83 Hughenden Lane, Glasgow G12 9XN, UK; bryan.jennett@ntlworld.com

\section{REFERENCES}

1 Jennett B, Plum F. Persistent vegetative state after brain damage. A syndrome in search of a name. Lancet 1972;i:734-7

2 Royal College of Physicians Working Group. The permanent vegetative state. J $R$ Coll Physicians Lond 1996:30:119-21.

3 Jennett B. The vegetative state. Medical facts, ethical and legal dilemmas. Cambridge: Cambridge University Press, 2002.

4 Multi-Society Task Force On PVS. Statement on medical aspects of the persistent vegetative state. N Engl J Med 1994;330:1499-508, 1572-9.

5 Andrews K, Murphy L, Munday R, et al. Misdiagnosis of the persistent vegetative state: retrospective study in a rehabilitation unit. $B M$ 1996;313:13-16. 
6 Giacino JT, Ashwal S, Childs N, et al. The minimally conscious state: definition and diagnostic criteria. Neurology 2002:58:349-53.

7 Ashwal S, Cranford R. The minimally conscious state in children. Semin Pediatr Neurol 2002; 9: 19-34.

8 Schiff ND, Ribary U, Moreno DR, et al. Residual cerebral activity and behavioura fragments can remain in the persistently vegetative brain. Brain 2002;125:1210-34.

9 Menon DK, Owen AM, Williams EJ, et al. Cortical processing in persistent vegetative state. Lancet 1999;352:200.

10 Schiff ND, Plum F. Cortical function in the persistent vegetative state. Trends $\mathrm{Cog} \mathrm{Sci}$ 1999:3:43-6.

11 Adams JH, Graham DI, Jennett B. The neuropathology of the vegetative state after an acute brain insult. Brain 2000;123:1327-38.
12 Jennett B, Adams JH, Murray S, et al. Neuropathology in vegetative and severely disabled patients after head injury. Neurology 2001;56:486-90

13 Ashwal S, Eyman RK, Call TL. Life expectancy of children in a persistent vegetative state. Pediatr Neurol 1994; 10:27-33.

14 Bernat JL. Questions remaining about the minimally conscious state. Neurology 2002;58:337-8.

15 Strauss DJ, Shavelle RM, Ashwal S. Life expectancy and median survival time in the permanent vegetative state. Pediatr Neurol permanent vegetalive

16 Andrews K. Managing the persistent vegetative state: early skilled treatment offers the best hope for optimal recovery. BM 1992;305:486-7.

17 Giacino JT. Sensory stimulation: theoretical perspectives and the evidence for effectiveness. Neurorehabil 1996:6:69-78.
18 Gillon R. Euthanasia, withholding life-prolonging treatment, and moral differences between killing and letting die. $J$ Med Ethics 1988;14:115-17.

19 Gillon R. Persistent vegetative state and withdrawal of nutrition and hydration. J Med Ethics 1993;19:67-8.

20 Wade DT. Ethical issues in diagnosis and management of patients in the permanent vegetative state. BM 2001;322:352-4.

21 Grubb A, Walsh P, Lambe N. Reporting on the persistent vegetative state in Europe. Med Law Rev 1998;6:161-210.

22 NHS Trust $A \vee$ Mrs M and NHS Trust B v Mrs $H$ [2001] All ER 801.

23 McLean SAM. Legal and ethical aspects of the vegetative state. J Clin Pathol 1999:52:490-3.

24 McLean SAM. Permanent vegetative state and the law. J Neurol Neurosurg Psychiatry 2001;71 (suppl 1):126-7. textbooks as recently as the 10 years ago. It so happens that the theoretical importance of neglect led to a disproportionate amount of basic research into this phenomenon. We are already seeing similar advances in theoretically derived and effective brain rehabilitation in a raft of other disorders, from aphasia to dyspraxia, and from dysexecutive syndrome to attentional deficits. ${ }^{5}$

As with all advances in health care, these treatments will require funds to be implemented. Rehabilitation has suffered in many parts of the world because it is labour intensive and because its evidence base has been lacking. Yet the costs of not treating the damaged brain in the most scientific and efficient way will be much greater in terms of greater dependency and lowered quality of life, particularly as a worldwide ageing population throws up more of these types of now increasingly ameliorable problems.

J Neurol Neurosurg Psychiatry 2002;73:357 et al (this issue, pp 412-9), ' we see clear evidence for the value of such promissory notes and the practical clinical benefits of grounding brain rehabilitation research in strong, theoretically driven cognitive neuroscience.

Spatial neglect following right cerebral stroke not only causes patients to behave as if the left half of the world does not exist; more practically it prevents them learning to walk again, renders them heavily dependent on carers, and massively reduces their quality of life. Schindler and his colleagues in Munich have shown that doing something quite implausible-applying a standard electromechanical vibrator to the left neck muscles of patients while they engage in visual search exercisesproduces marked and enduring clinical and real life benefits.

This treatment arose out of basic research into the brain mechanisms of sensory integration and higher level perception. Neglect patients were studied because of what their damaged brains revealed about the functional architecture of the intact brain. Neck vibration was used purely because of its known effects on the body's normal coordinate frame of reference according to which sensory inputs and motor outputs are integrated. In neglect, not only is this egocentric reference frame biased to the right, but also the neck vibration can temporarily correct this imbalance. What we see in the Munich paper is that, when combined with systematic visual search training, and when systematically applied for 15 treatment sessions over three weeks, temporary effects become long lasting and hence therapeutically important.

Similarly impressive results in neglect rehabilitation have been obtained with another new cognitive neuroscience derived treatment-prism adaptation training ${ }^{2}$ - and there are several other counterintuitive but potent interventions emerging from modern cognitive neuroscience. One such example is limb activation training, which induces neglect patients to make small movements with the left side of their body in left hemispace in order to improve their visual attention to the left ${ }^{3}$ : acute neglect patients receiving this minimally labour intensive additional treatment are discharged from hospital on average 28 days earlier than patients who do not receive it. ${ }^{4}$

But is there something special about unilateral neglect? Not at all: neglect was considered untreatable in some standard 


\section{We are how we eat?}

\section{Mummery}

\section{The underlying neural substrates of eating behaviours}

T he two most common dementias in the presenium are Alzheimer's dementia (AD) and frontotemporal dementia (FTD). Early and accurate diagnosis is critical for prognosis, consideration of genetic issues, and appropriate therapeutic intervention. Recognition of associated behavioural disturbance is paramount in order to address the enormous caregiver burden with sufficient resources. However, early recognition is still fraught with difficulty, exacerbated by a lack of systematic studies. As conventional cognitive assessment can fail to reveal significant behavioural deficits early in disease, it is imperative to develop new and sensitive tools, such as that described in the paper by Ikeda et al (this issue, pp 371-6). ${ }^{1}$

Previous work has shown that, although there are marked behavioural differences between FTD and AD, changes in eating preference are one of the few behaviour variables that reliably separate the two groups. ${ }^{2}$ Within FTD, in spite of the common perception that there is less behavioural disturbance in semantic dementia (SD or temporal variant FTD) than in frontal variant (fv) FTD, analysis has shown comparable prevalence. ${ }^{2}$ It is possible that behavioural changes in SD have been overlooked in the past due to the profound linguistic deficits seen. However, there may be subtle qualitative differences between fvFTD and SD behaviour. One study found more indiscriminate eating behaviour, such as gluttony, in fvFTD whereas SD was characterised by increased selectivity and food fads. ${ }^{3}$

Ikeda et al performed a detailed examination of eating behaviour change in patients with fvFTD, SD, and AD, using a prospective caregiver questionnaire. ${ }^{1}$ Frequency of eating behaviour change was higher in both types of FTD than in $\mathrm{AD}$, in particular changes in food preference and eating habits. They found remarkably little qualitative difference between fvFTD and SD. Although this seems to contradict previous findings, ${ }^{3}$ examination of their data reveals that faddy food behaviours were more frequent in SD and overeating more frequent in fvFTD, though differences were not significant. Increased appetite was associated with SD and fvFTD, whereas anorexia was associated with $\mathrm{AD}$. The finding of early onset dysphagia in some $\mathrm{AD}$ patients emphasises the importance of questioning caregivers about swallowing problems. Ikeda et al also examined the order of development of eating behaviour change. The SD patients showed a remarkably consistent pattern of change. In contrast, the pattern was unclear in $\mathrm{AD}$.

From a theoretical viewpoint, this study raises interesting possibilities. The ventral frontal lobe, temporal pole, and amygdala are damaged in both fvFTD and $\mathrm{SD}^{4}$; the finding of common eating behaviour changes in the two groups supports the hypothesis that these areas are implicated in eating disorders. The consistency in symptom development in SD suggests that serial imaging could be used to shed light on the structural and functional brain changes associated with symptom progression. In this way behavioural descriptions allied to functional studies may additionally inform us as to the underlying neural substrates of eating behaviours. From a clinical viewpoint, Ikeda et al provide an increased awareness of specific eating behaviour abnormalities, encouraging early intervention and accurate therapeutic monitoring of new treatments such as the SSRIs. The attention to detail in this new tool should pay dividends in understanding normal and abnormal eating patterns.

J Neurol Neurosurg Psychiatry 2002;73:358

\section{Author's affiliation}

Dr C Mummery, Department of Neurology, Kings College Hospital, Denmark Hill, London SE5, UK; CathMummery@aol.com

\section{REFERENCES}

1 Ikeda M, Brown J, Holland AJ, et al. Changes in appetite, food preference, and eating habits in frontotemporal dementia and Alzheimer's disease. J Neurol Neurosurg Psychiatry 2002;73:371-6.

2 Bozeat S, Gregory CA, Lambon Ralph MA, et al. Which neuropsychiatric and behavioural features distinguish frontal and temporal variants of frontotemporal dementia from Alxheimer's disease? J Neurol Neurosurg Psychiatry 2000;69:178-86.

3 Snowden JS, Bathgate D, Varma A, et al. Distinct behavioural profiles in frontotemopral dementia and semantic dementia. J Neurol Neurosurg Psychiatry 2001;70:323-32.

4 Mummery CJ, Patterson K, Wise RSF, et al. A voxel based morphometry study of semantic dementia: the relationship between temporal lobe atrophy and semantic dementia. Ann Neurol 2000;47:36-45.

\section{Infection and multiple sclerosis - a new hypothesis?}

\section{G J Stewart}

\section{Hypothesis of a sexually transmitted environmental component should be treated with care}

$\mathrm{T}$ here is general agreement that multiple sclerosis is the result of both environmental and genetic factors. In the paper by Hawkes ${ }^{1}$ (this issue, pp 439-43) a hypothesis is proposed that the environmental component is an infectious agent, transmitted sexually. His thesis is based on a new look at old data; it has not been stimulated by recent discovery.
Evidence for an environmental factor in multiple sclerosis has come from studies of migrating populations, small epidemics and variation of prevalence with latitude. Many of these studies are cited by Hawkes. The possibility that the environmental factor is a transmissible infection was reviewed in detail by Kurtzke $^{2}$ in 1993, who proposed that the MS agent is an as yet unidentified retrovirus that results in widespread asymptomatic infection in early adolescence. Hawkes' extension of the Kurtzke $^{2}$ hypothesis to a sexually transmitted infection (STI) with a neurotropic agent appears to rely on his interpretation of the higher prevalence of MS in women, the low prevalence in native populations isolated from white males, a proposed correlation between 
sexual permissiveness and MS prevalence and with aspects of other diseases due to a known sexually transmissible agent, particularly HTLV-1.

Whilst this succinct analysis and the accompanying table represent a scholarly and, in most parts, balanced effort, there are substantial difficulties with this hypothesis that can't be overlooked. Adoption studies are not, as claimed, compatible with the STI hypothesis; the large Canadian MS adoptee study ${ }^{3}$ indicated that familial clustering is explained predominantly, if not exclusively, by genetic susceptibility. In the same population, there is convincing evidence that MS spouses are not at increased risk ${ }^{4}$ and some migration studies suggest an environmental factor acting well into adult years. ${ }^{5}$ Hawkes assumes close contact to mean sexual contact in the MS clusters without evidence. The explanation for the low MS prevalence in the isolated populations highlighted by Hawkes is more plausibly genetic. The lack of evidence for transmission of MS risk vertically, by breast milk, or blood transfusion represents a major difference between the epidemiology of MS and known retroviral infections such as HTLV-1 and HIV. The data cited with respect to drug use are not interpretable.

There is also an issue of particular concern. People living with MS are often, fortunately, well connected to the world literature. The suggestions that susceptibility may be linked to sexual permissiveness or that childhood MS could result from child abuse have the potential to cause harm, unless it is made clear that they are pure speculation, based on interpretation of data collected for different purposes.

Is the case, therefore, in support of an STI in MS aetiology sufficient to merit the case control studies needed to test this hypothesis? It would appear not, but readers will make their own judgements. A more prudent approach is perhaps to await the discovery of new infectious organisms, including STI, and to assess directly any relationship to MS as has occurred for HTLV-1, HIV, and other agents.

J Neurol Neurosurg Psychiatry 2002;73:358359

\section{Author's affiliation}

G J Stewart, Institute for Immunology \& Allergy Research, Westmead Millennium Institute, Westmead Campus, University of Sydney, Westmead NSW 2145, Australia;

stewartg@westgate.wh.usyd.edu.au

\section{REFERENCES}

1 Hawkes, $\mathrm{CH}$. Is multiple sclerosis a sexually transmitted infection? J Neurol Neurosurg Psychiatry 2002;73:439-43.

2 Kurtzke, J. Epidemiologic evidence for multiple sclerosis as an infection. Clin Microbiol Rev 1993:6:382-27.

3 Ebers GC, Sandovnick AD, Risch NJ, and the Canadian Collaborative Study Group. A genetic basis for familial aggregation in multiple sclerosis. Nature 1995;377:150-1.

4 Ebers GC, Yee IML, Sandovick AD, Duquette P and the Canadian Collaborative Study Group. Conjugal multiple sclerosis: population-based prevalence and recurrence risk in offspring Ann Neurology 2000;48:927-31

5 Hammond SR, English DR, McLeod JG. The age-range of risk of developing multiple sclerosis: evidence from a migrant population in Australia. Brain 2000;123:968-74.

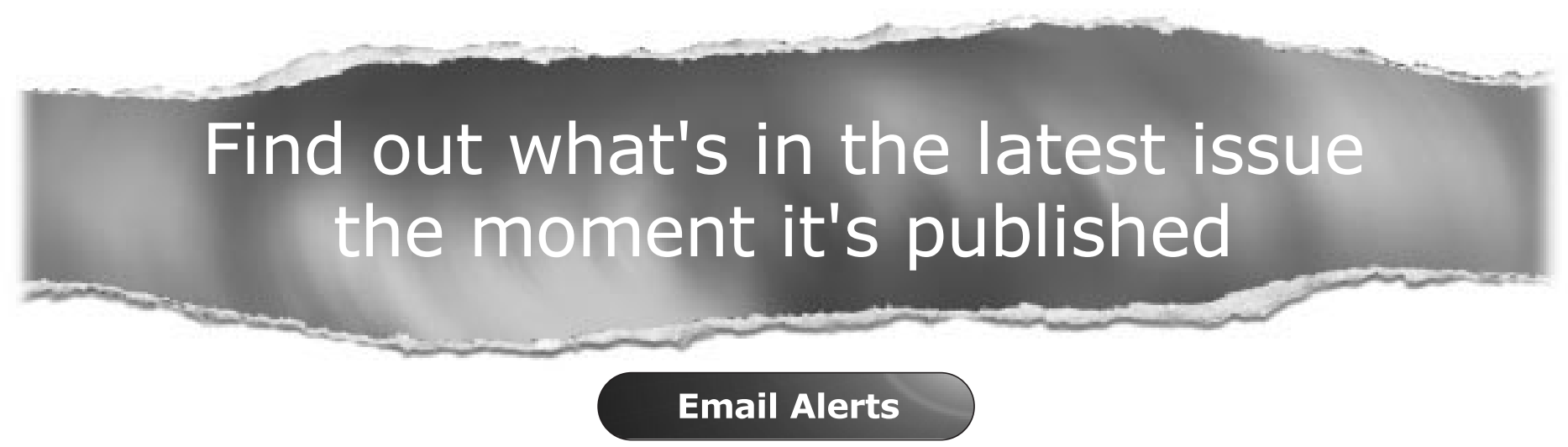

Sign up to receive the table of contents by email every month. You can select from three alerts: Table of Contents (full), TOC Awareness (notice only); Journal of Neurology, Neurosurgery, and Psychiatry related announcements.

www.jnnp.com 